entire globe, but there needs to be a system in place to ensure their images are readily available to everyone who needs them. Brazil has set an important precedent by making its Earth-observation data available, and the rest of the world should follow suit. This is more than a matter of common courtesy. It will foster the kinds of checks and balances and independent analysis that must necessarily underpin a viable carbon market.

And the international community needs to start thinking about the next step: how to encourage good forest stewardship. As it stands, nations such as India and Costa Rica are in the odd position of receiving little or no benefit from a market in carbon credits precisely because they have been able to control deforestation. And if illegal deforestation were to come to a halt, then those nations benefiting from the carbon market would see that source of income dry up, creating the same pressures that caused the problem in the first place.

True, dealing with standing forests will be tricky; no one wants to create a permanent welfare programme for the tropics. Nevertheless it is vital that the issue is tackled. This is essentially what the delegates agreed to do last December at the United Nations climate-change

conference in Bali, and their decision was a wise one. As long as the international community is playing with the architecture of a carbon economy, it should explore new and creative ways to build in 'ecosystem services' such as biodiversity and coastal protection. Bear in mind that the alternative to putting an economic value on these intangibles is implicitly to set their value at zero.

One of the oddly positive effects of global warming is that it has given the world the opportunity to build a more comprehensive and inclusive

"Global warming has
given the world the
opportunity to build a more comprehensive and inclusive economic model."

economic model by forcing all of us to grapple with our impact on the natural environment. We are entering a phase in which new ideas can be developed, tested, refined and rejected as necessary. If we find just one that can beat the conventional economic measure of gross domestic product, and can quantify some of the basic services provided by rainforests and other natural ecosystems, it will more than pay for itself.

\section{On message, off target}

\section{Official advice on vaccination is too often poorly transmitted.}

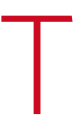

he cause of science-based policy was not helped late last month when the presumptive Republican candidate for the US presidency, John McCain, promoted the discredited notion that a preservative in vaccines causes autism. Such statements have power, as Anna Pearce and her colleagues remind us in the online edition of the British Medical Journal (A. Pearce et al. Br. Med. J. doi:10.1136/ bmj.39489.590671.25; 2008). They find that, following the 1998 scare in Britain, when autism was baselessly linked to the triple vaccination for measles, mumps and rubella, uptake by parents, having bottomed out at $79 \%$, has now climbed to $89 \%$ - but still falls below the $95 \%$ level required for herd immunity.

The paper, valuable in its analysis of parents' decision-making, emphasizes the need to provide "evidence based information ... tailored to respond to particular concerns, questions, and beliefs of different groups". Unfortunately, it seems that official sources are not following that advice.

Consider, for example, a separate study of the cognitive frameworks that US parents use to absorb information about vaccination, carried out by Baruch Fischhoff, an authority on risk perception, and his colleagues at Carnegie Mellon University in Pittsburgh, Pennsylvania (J. S. Downs et al. Vaccine doi:10.1016/j.vaccine.2008.01.011; 2008). The researchers conducted phone interviews with parents in three US cities - Kansas City, Philadelphia and Eugene - and did in-depth interviews with parents selected by a market-research company for diversity in race, background and vaccination attitudes. The number was small $-30-$ and the authors rightly emphasize that the study is preliminary rather than conclusive. But the results are suggestive, and consistent enough to make any sensible policy-maker think.
Working with officials at the Centers for Disease Control and Prevention (CDC) in Atlanta, Georgia, the team identified 24 possible conceptual variables that might feature explicitly or implicitly in parents' decisions whether or not to have their child vaccinated. These include 'public-health credibility', 'benefit of vaccinating, 'herd immunity', 'information,' 'personal values', 'vaccine safety research' and 'risk of vaccinating.'

The parents, all with children aged between 18 and 23 months, were interviewed individually in a way that explored their 'mental model' of vaccination - that is, their beliefs about these concepts and the relationships between them. The parents were also presented with official pro-vaccination communications from the $\mathrm{CDC}$ and unofficial antivaccination communications, along with questions that explored their trust in both. By analysing the parents' responses for each communication, the authors were able to map how it influenced their mental models, and ultimately their decision whether to vaccinate.

The authors' conclusion is that anti-vaccination communications play on a much richer field of considerations in parents' heads than the official communications. The latter rely on science-based reassurance - and, indeed, were received by the parents with a relatively high degree of trust. But they tell a much less comprehensive and connected story than the anti-vaccination communications, which in some cases ended up having a correspondingly higher degree of influence.

Not surprisingly, many parents spoke of the Internet as a key source of advice. The authors explored how the parents were using the Internet and then conducted similar searches to see what they would find. They concluded that more than $90 \%$ of the parents would encounter anti-vaccination advice among the top 10 returns from search engines, with such results often ranking higher than official websites.

John McCain's comments won't have helped US public health but neither, it seems, do most official communications. The CDC and others sources of health advice need to be much more sophisticated in how they communicate if vaccination myths are to be successfully countered. 